\title{
LITERATUR
}

\section{Mehr als ein Nebenschauplatz? Neue Beiträge zur Analyse der Wahlen zum Europäischen Parlament}

\author{
Frank Wendler*
}

Die Wahlen zum Europäischen Parlament werden seit der ersten Direktwahl im Jahr 1979 als nationale Nebenwahlen (oder in der ursprünglichen Begriffsprägung von Karlheinz Reif und Hermann Schmitt als ,second-order national elections"1) charakterisiert. Auch nach der letzten Europawahl im Jahr 2009 erscheint unbestritten, dass die wichtigsten Aussagen der Nebenwahlthese trotz der gewachsenen Kompetenzen des Parlaments und der zunehmenden politischen Brisanz vieler europapolitischer Themen weiterhin Gültigkeit besitzen: Demnach sind Europawahlen durch eine im Vergleich zu nationalen Hauptwahlen geringe Wahlbeteiligung, die Nachrangigkeit von europapolitischen gegenüber nationalstaatlichen Themen und Akteuren sowie relativ hohe Stimmenanteile kleinerer und oppositioneller Parteien charakterisiert. Sie werden damit primär als von nationalen Wahlzyklen bestimmte Protestvoten gegen amtierende Regierungsparteien auf nationaler Ebene interpretiert. Trotzdem ist in neueren Forschungsbeiträgen $\mathrm{zu}$ diesem Thema das Interesse erkennbar, nach Ergänzungen zum fast schon reflexartig angeführten Nebenwahlmodell zu suchen und Fragen anzusprechen, die von diesem Modell ignoriert oder nicht ausreichend geklärt werden. Dies betrifft drei Punkte. Erstens stellt sich für die Wahlforschung die Frage, ob der hohe Grad an Wahlenthaltung mit Einstellungen der Wähler(-innen) gegenüber der europäischen Integration oder den Politiken der Europäischen Union in Verbindung gebracht wer-
Jürgen Mittag (Hrsg.): 30 Jahre Direktwahlen zum Europäischen Parlament (1979-2009). Europawahlen und EP in der Analyse, Nomos: BadenBaden 2011, ISBN 978-3-8329-6363-7; 372 Seiten, $49,00 €$.

Robert Harmsen/Joachim Schild (Hrsg.): Debating Europe. The 2009 European Parliament Elections and Beyond, Band 71 der Schriftenreihe des Arbeitskreises Europäische Integration e.V., Nomos: Baden-Baden 2011, ISBN 978-38329-5807-7; 280 Seiten, 49,00 €.

Michaela Maier/Jesper Strömbäck/Lynda Lee Kaid (Hrsg.): Political Communication in European Parliamentary Elections, Ashgate: Farnham/ Burlington 2011, ISBN: 978-1-4094-1132-1; 268 Seiten, 73,99€.

den kann und somit als Ausdruck einer gewachsenen Europaskepsis oder eher als Teil eines desinteressierten ,permissiven Konsensus ‘ zu deuten ist. Zweitens bleibt für die Parteien- und politische Kommunikationsforschung die Frage offen, welche Strategien zur Verknüpfung zwischen der Europapolitik und innenpolitisch relevanten Themen eingesetzt, in welcher Weise also Europawahlen von Parteien und Regierungen zu nationalen Nebenwahlen umfunktioniert werden. Drittens entdeckt die diskursiv ausgerichtete Forschung zur ,Europäisierung“ politischer Systeme inzwischen auch die Europawahlen als Forschungsfeld. Im Zentrum des Forschungsinteresses steht hier die vergleichende Untersuchung der Frage, welche Perzeptionen der

* Dr. Frank Wendler, Institut für Politikwissenschaft, Goethe-Universität Frankfurt am Main.

1 Vgl. Karlheinz Reif/Hermann Schmitt: Nine Second-Order National Elections - A conceptual Framework for the Analysis of European Election Results, in: European Journal of Political Research 1/1980, S. 3-45. 
europäischen Integration in den Mitgliedstaaten vorherrschen und in welchem Maße sie zum politischen Streitobjekt werden. In diesem Zusammenhang hat das mittlerweile in der Integrationsforschung geläufige Schlagwort der Politisierung auch die Forschung zu Europawahlen erreicht.

Zeitlicher Rückblick: Europawahlen als enttäuschter Demokratisierungsansatz?

Diese Fragestellungen spiegeln sich in unterschiedlicher Weise in den hier besprochenen drei Sammelbänden, die vor Kurzem anlässlich der Wahl des Jahres 2009 erschienen sind. Ein breit gefächertes Spektrum von Beiträgen findet sich in einem von Jürgen Mittag herausgegebenen Sammelband, ${ }^{2}$ der durch seinen Titel ,30 Jahre Direktwahlen zum Europäischen Parlament (1979-2009)" schon das Interesse seiner Autor(-innen) an einer ausführlichen Wiedergabe der bisherigen Entwicklung des Parlaments und der Europawahlen andeutet. Wenig überraschend finden sich auch in diesem Band zahlreiche Bezugnahmen auf das ,second-order'-Modell als dem wichtigsten Interpretationsrahmen der Europawahlen. Davon ausgehend identifiziert der Herausgeber in seiner Einleitung aber das Vorhaben einer diachronen Betrachtung der Entwicklung der Europawahlen sowie eine Bestandsaufnahme der Bedeutung und Grenzen ihrer legitimatorischen Kraft als Lücke der bisherigen Forschung. Damit wird ein triftiges Argument in Bezug auf die Neutralität der Nebenwahlthese gegenüber der dynamischen Entwicklung der Europäischen Union als politisches System angesprochen: Auch wenn die Einstufung der Europawahlen als Nebenwahl im Gesamtbild plausibel erscheint, wird damit der überaus unterschiedliche Kontext der politischen und institutionellen Entwicklung des Europäischen Parlaments und der gesamten Europäischen Union zum Zeitpunkt der verschiedenen Wahlgänge überblendet.
Dieser Zugang wird im Rahmen des Sammelbandes in fünf Schritten entwickelt. Ein erster Block aus drei sehr grundständig wirkenden Kapiteln versucht eine Einordnung der Repräsentationsbasis und institutionellen Funktion des Parlaments und diskutiert die These des europäischen Demokratiedefizits sowie der Einstufung der Europawahlen als Nebenwahlen.

Der zweite Teil wendet sich dann stärker dem Ansatz einer historischen Rekonstruktion zu und legt in zwei Kapiteln den Weg zur Direktwahl des Europäischen Parlaments von den 1950er Jahren bis zu den entscheidenden Beschlüssen der 1970er Jahre dar. Zwei weitere Kapitel nehmen eine anschließende Bewertung und Einschätzung der ersten Direktwahl im Jahr 1979 vor.

Der dritte Teil unternimmt in fünf Teilkapiteln eine ausführliche Darstellung der bisherigen sieben Wahlgänge, ergänzt durch eine Längsschnittanalyse zur Erklärung der Wahlbeteiligung bei den Europawahlen. Dieser Beitrag verneint dabei einen Zusammenhang zwischen Enthaltung bei Europawahlen und negativen Haltungen der Nicht-Wähler(-innen) gegenüber der europäischen Integration. Die vier Einzelkapitel zur ersten Direktwahl sowie den jeweils zwei Wahlen in den nachfolgenden drei Jahrzehnten gehen einzeln pro Wahl auf die wesentlichen Wahlkampfthemen, die Wahlergebnisse im Verhältnis zu den Kräfteverhältnissen auf nationaler Ebene und die Fraktionsbildung im Europäischen Parlament ein. Diese Darstellungen sind systematisch und informativ geschrieben, wirken aber hochgradig deskriptiv und räumen den aktuelleren Wahlen von 2004 und 2009 nicht mehr Raum ein als den länger zurückliegenden Wahlen der 1980er und 1990er Jahre. Auf eine echte Längsschnittsanalyse wird verzichtet.

2 Jürgen Mittag (Hrsg.): 30 Jahre Direktwahlen zum Europäischen Parlament (1979-2009). Europawahlen und EP in der Analyse, Baden-Baden 2011. 
Dieses Element findet sich stärker in einer Analyse von Plakaten aus Wahlkämpfen im Zeitraum zwischen 1979 und 2009, die zusammen mit einer Untersuchung von Parteitagen zur Europawahl den vierten Teil des Buches zur Vermittlung und Inszenierung von europapolitischen Positionen der Parteien bilden.

Den interessantesten Abschnitt des Buches bildet der abschließende fünfte Teil, der Perspektiven einer Parlamentarisierung und Politisierung der Europäischen Union diskutiert. Die vier Kapitel in diesem Teil diskutieren die Neuerungen des Vertrags von Lissabon, die Weiterentwicklung der europäischen Parteienstrukturen am Beispiel der europäischen Sozialdemokraten, die Rolle des Parlaments im Institutionengefüge der Europäischen Union und schließlich existierende Vorschläge zur Reform des Wahlsystems zum Europäischen Parlament. Sehr weit geht diese Darstellung zwar nicht über den Forschungsstand zur Entwicklung des Europäischen Parlaments und der europäischen Parteiendemokratie hinaus. Allerdings gelingt den Autor(-innen) eine interessante und anregende Zusammenfassung und Bündelung vorhandener Vorschläge und Thesen zur Zukunft der parlamentarischen Demokratie in Europa. Auch insgesamt liegt der Wert dieses Sammelbandes eher in der breit angelegten Rekonstruktion der bisherigen Entwicklung der Europawahlen und damit verbundener politikwissenschaftlicher Debatten als in der Erschließung neuer analytischer oder theoretischer Zugänge. Insgesamt bleibt vor allem die These stehen, dass die Einführung der Direktwahlen zum Europäischen Parlament einen im damaligen Zeitkontext mit hohen Erwartungen befrachteten, letztlich aber enttäuschenden Ansatz zur politischen Öffnung und Demokratisierung des politischen Systems der Europäischen Union darstellt. $\mathrm{Ob}$ mit den abschließend vorgestellten Ansätzen zur Stärkung des Parteienwettbe- werbs bei Europawahlen ein Durchbruch zu stärker europapolitisch geprägten Wahlkämpfen und eine stärkere Bindung an die europäischen Wahlbürger(-innen) erzielt werden können, muss dabei fraglich bleiben.

\section{Wahlen zum Europäischen Parlament als Arena europapolitischer Diskurse}

Ausdrücklich an einer Erweiterung der Nebenwahlthese ausgerichtet ist der von Robert Harmsen und Joachim Schild herausgegebene englischsprachige Sammelband zur Wahl im Jahr 2009. ${ }^{3}$ In ihrer Einleitung vermerken die beiden Herausgeber, das , second-order'-Modell verdecke Fragen nach der Rolle von Europawahlen als Arena des parteipolitischen Wettbewerbs und die Untersuchung der verschiedenen nationalen Europadiskurse und ihrer Umstrittenheit in der öffentlichen Debatte. Als gemeinsamen Analyserahmen stellen sie den einzelnen Länderberichten die Unterscheidung von vier thematischen Kategorien der europapolitischen Debatte voran: Unterschieden werden kritische Diskurse in Bezug auf das politische Projekt der europäischen Integration, seine sozialen und ökonomischen Auswirkungen und seine Vereinbarung mit ideellen Konzepten nationaler Identität sowie Diskurse politischer Eliten zur Legitimierung des Projekts der europäischen Integration. Auch wenn diese Kategorisierung als thematisch konzipierte Unterscheidung nicht ganz schlüssig ist, gelingt den Autor(-innen) des Bandes in den nachfolgenden Einzelbeiträgen damit eine aufschlussreiche Systematisierung der europapolitischen Debatten in den betrachteten Mitgliedstaaten. Die Länderkapitel behandeln nacheinander die fünf Gründungsstaaten der Europäischen Gemeinschaft außer Luxemburg, vier als „West European ,latecomers “" bezeichnete Staaten (Dänemark, Großbritannien, Spanien und Finnland) sowie vier der seit 2004 beigetretenen Staaten (Tschechische Republik, Lettland, Slowakei und Rumänien). Die auch von namhaften Autor(-innen)

3 Robert Harmsen/Joachim Schild (Hrsg.): Debating Europe. The 2009 European Parliament Elections and Beyond, Baden-Baden 2011. 
wie Siegfried Schieder, Hans Vollaard, José M. Magone, Tapio Raunio und Karen Henderson verfassten Länderkapitel sind allesamt klar und systematisch geschrieben und enthalten eine sorgfältige Dokumentation der Parteipositionen, politischen Debatten und Wahlergebnisse. Als Einzelfallstudien erscheinen diese für alle an den europapolitischen Debatten der einzelnen Länder Interessierten allemal lesenswert.

Im abschließenden Überblick stellt sich aber die Frage, inwieweit aus der Sammlung dieser Studien allgemeine Erkenntnisse über die Entwicklung der Europawahlen gezogen werden können. Die Herausgeber versuchen dies in ihrem abschließenden Kapitel zunächst durch den Abgleich von Daten zu den europapolitischen Haltungen der Bürger(-innen) und Parteien der untersuchten Länder. Durch die quantitative Zugangsweise und die relativ simple Auswertung der Daten durch deskriptive Statistik wirkt dieser Ansatz im Rahmen der qualitativen Methodik des Bandes etwas unpassend. Davon ausgehend kristallisieren sich im Resümee der Herausgeber aber einige sehr interessante Beobachtungen aus dem Vergleich der europapolitischen Diskurse der untersuchten Länder heraus. So erscheinen die klassischen, integrationsfreundlichen Europadiskurse in den fünf betrachteten Gründungsstaaten überfordert und weichen inhaltlich veränderten sowie insgesamt auch politisch umstritteneren öffentlichen Debatten. Das Ergebnis ist eine $\mathrm{Zu}-$ nahme politischer Kritik an der europäischen Integration aus dem politischen Mainstream und das Entstehen einer pragmatischeren und stärker utilitaristisch geprägten Haltung zur europäischen Integration, vor allem in Deutschland, Italien und den Niederlanden. Auch in Frankreich wird das Zerfallen der diskursiv artikulierten Idee einer „Europe puissance“ als Grundlage für die Projektion außenpolitischer Interessen und Werte der fünften Republik beobachtet. Demgegenüber werden die europapolitischen Debatten der ,neuen“ Mitgliedstaaten als Beleg für die diskursive Externalisierung der europäischen Integration in den vier betrachteten Ländern gedeutet. In diesem Sinne erscheint die Politik der Europäischen Union als Quelle politischer Handlungsanreize durch das Angebot von finanziellen Ressourcen, politischen Lösungsansätzen und gesellschaftlicher Modernisierung, aber nicht als internalisierter Bestandteil des politischen Wettbewerbs zwischen den Parteien. Klare Unterschiede werden davon ausgehend vor allem zwischen den relativ integrationsfreundlichen Debatten Rumäniens, der Slowakei und Lettlands sowie den deutlich stärker europaskeptisch ausgerichteten Debatten in der Tschechischen Republik konstatiert. Keine klare Gemeinsamkeit findet sich in Bezug auf die westeuropäischen ,latecomer", was allerdings angesichts der sehr unterschiedlichen Zugänge der untersuchten Staaten zur Europäischen Union kaum erstaunen kann. Lesenswert erscheint angesichts der neueren Entwicklung vor allem das Kapitel zu Finnland, in dem der elektorale Durchbruch der „Wahren Finnen“ als Ausdruck der auch in den Gründungsstaaten konstatierten ,Normalisierung' der politischen Debatte im Zusammenwirken mit der hohen Diskrepanz zwischen Eliten- und Wählerpositionen gedeutet wird.

Insgesamt überzeugt der Sammelband mit seiner These, dass Europawahlen trotz der nicht infrage gestellten Gültigkeit des ,second-order'-Modells als Arena für die Entfaltung europapolitischer Debatten und Diskurse ernster genommen werden sollten. Die Unterscheidung der vier Diskurstypen wird dabei nicht in allen Beiträgen strikt eingehalten, erscheint aber als zielführender Ansatz zum systematisierenden Vergleich der Debatten in den 13 untersuchten Mitgliedstaaten der Europäischen Union. Gegenüber der Vielzahl quantitativ angelegter Wahlanalysen ist der Band vor allem für die an vergleichender qualitativer Diskursanalyse interessierte Europaforschung als sehr wertvolle Ergänzung der Literatur anzusehen.

\section{Die europäische Integration als Thema der} politischen Kommunikation

Methodisch aufwändiger, aber auch inhaltlich disparater wirkt der von Michaela Maier, Jesper Strömbäck und Lynda Lee Kaid heraus- 
gegebene Sammelband zur politischen Kommunikation bei Europawahlen. ${ }^{4}$ Auch hier erfolgt der Einstieg über eine Kritik des ,secondorder'-Modells: Interessanter als der Hinweis auf die Nachrangigkeit von Europawahlen sei die vergleichende Untersuchung systembedingter Unterschiede und thematischer Verknüpfungen zwischen verschiedenen politischen Ebenen in der politischen Kommunikation. Dieser an sich interessante Fokus auf die komparative Analyse kommunikativer Strategien von Parteien und Medien im Kontext der Europawahlen wird aber in den Beiträgen des Sammelbandes nicht konsequent durchgehalten. In diesem Sinne unpassend wirkt die gleich zu Beginn eingefügte, sehr weit ausholende Darstellung der rechtlichen und institutionellen Grundlagen des Europäischen Parlaments und der Europawahlen seit der Gründung der parlamentarischen Versammlung im Jahr 1952 und der 1979 erfolgten Einführung von Direktwahlen. Auch die sonst lesenswerten Kapitel zur Anwendung des ,second-order'-Modells auf die Wahlen 2009 von Michael Marsh und zur Analyse der Wahlbeteiligung in den Wahlen von 1979 bis 2009 von Gilg U. H. Seeber und Markus Steinbrecher (die ebenfalls eine Verknüpfung zwischen Wahlenthaltung und Einstellungen zur Europäischen Union verneinen) passen somit nicht zum thematischen Schwerpunkt des Buches.

Anders als im eben besprochenen Band präsentieren die Einzelbeiträge des Buches aber nicht nur drei Fallstudien, sondern auch drei Zweiervergleiche und sechs breit angelegte Vergleichsstudien, die eine große Menge von selbst erhobenen Primärdaten aus allen 27 Mitgliedstaaten zusammentragen. Die meisten Beiträge präsentieren zum Teil sehr umfangreiche Daten aus der quantitativen Codierung von Zeitungsbeiträgen und Fernsehspots zur Wahl 2009, die sich auf die thematische Struktur der Beiträge, den Grad der Personalisierung von Berichten, die angesprochene politische Ebene (im Vergleich zwischen mitgliedstaatlicher und supranationaler Politik), das gewählte journalistische Format, erkennbare Wertungen der europäischen Integration und den Stil der politischen und journalistischen Darstellung (etwa im Vergleich von ,horse race" versus thematischen Beiträgen) beziehen.

Besonders lesenswert erscheint ein Vergleich der Professionalisierung von Kampagnen in Schweden und Finnland (Kapitel 3), ein Vergleich der thematischen Darstellung der Europawahlen zwischen Medien und Parteien am Beispiel Portugals (Kapitel 7) und die Analyse kommunikativer Frames in Bezug auf die Darstellung der Europäischen Union und der Positionen politischer Parteien in den Medien (Kapitel 10 und 11). Etwas irritierend an diesen und zwei weiteren Kapiteln mit breit angelegten Fallvergleichen ist, dass jeweils sehr viele, nämlich zwischen sieben und 13 Personen als Autor(-innen) des Beitrags genannt werden. Hier wäre eine Klärung wünschenswert gewesen, ob die Kapitel tatsächlich aus einer konzeptionellen Zusammenarbeit aller beteiligten Verfasser(-innen) hervorgegangen sind oder die meisten Beteiligten vor allem Daten zu Beiträgen beigesteuert haben, die von den als Erstautor(-innen) genannten Herausgeber(-innen) federführend entwickelt worden sind. Ebenfalls 13 Verfasser(-innen) hat das abschließende Kapitel, in dem der interessante Versuch eines experimentellen Tests zur Wirkung von Wahlkampfmaterial mit unterschiedlichen thematischen Schwerpunkten und Wertungen auf europapolitische Einstellungen von fünf Testgruppen aus jeweils 50 Personen dargestellt wird. Dieser und viele übrige Beiträge im Buch verdeutlichen den Anspruch der im Sammelband vertretenen Autor(-innen-)gruppe, durch die vergleichend angelegte Erhebung einer großen Menge von Primärdaten zur systematischen Durchleuchtung der Struktur politischer Debatten zum Europawahlkampf beizutragen. Die einzelnen Kapitel versammeln dabei eine große Anzahl gut aufbereiteter Auswertungen, die besonders für Leser(-innen)

4 Michaela Maier/Jesper Strömbäck/Lynda Lee Kaid (Hrsg.): Political Communication in European Parliamentary Elections, Farnham/Burlington 2011. 
mit einem Interesse an quantitativen und mit relativ hoher Fallzahl angelegten vergleichenden Studien zur Themenstruktur von Debatten bei Europawahlen interessant sein dürften.

Insgesamt bleibt aber die Aussage des Buches gegenüber der Nebenwahlthese - abgesehen von einer Vielzahl von thematisch relativ spezifischen Einzelbeobachtungen und dem Nachweis deutlicher Unterschiede in der inhaltlichen Struktur der politischen Kommunikation in den EU-Mitgliedstaaten - etwas unklar. Dazu trägt offenkundig die Tatsache bei, dass kein gemeinsames Analysemodell als Klammer für die unterschiedlichen Beiträge entwickelt wird. Das Buch bietet damit einen reichen Fundus an empirisch fundierten Erkenntnissen über die Entwicklung der politischen Kommunikation bei Europawahlen, es mangelt aber im Gesamtbild an einer zusammenfassenden Systematisierung, Einordnung und theoretischen Fundierung der gesammelten Erkenntnisse. Vor allem durch die systematische ErschlieBung der von den Medien und Parteien ausgehenden Kommunikation stellt der Band aber eine durchaus wertvolle Ergänzung des Forschungsstandes zu Europawahlen dar.

Fazit: Ansatzpunkte für eine Ergänzung der Nebenwahlthese

Insgesamt spiegelt sich in allen drei Neuerscheinungen das gewachsene Interesse der
Europaforschung an der Untersuchung des Parteienwettbewerbs und öffentlicher Debatten im Kontext der Europäischen Union. Die inzwischen weithin beachtete These einer Politisierung der europäischen Integration wird dabei mit Blick auf die Europawahlen der jüngeren Vergangenheit von keinem der drei Bände in umfassender Weise bestätigt: Ganz überwiegend sind politische Debatten und Wahlkämpfe vor Europawahlen vom Vorrang nationalstaatlicher Themen und einer relativ geringen Intensität der Parteikampagnen und Berichterstattung sowie einer gegenüber nationalen Wahlen geringen Polarisierung zwischen den Parteien geprägt. Es wird aber deutlich, dass Europawahlen kein reiner Nebenschauplatz nationaler Politik, sondern eine Arena für die Thematisierung national und parteipolitisch eingefärbter Sichtweisen auf die europäische Integration sind und somit viel über die Entwicklung der kommunikativen beziehungsweise diskursiven Dimension des Integrationsprozesses im Kontext der unterschiedlichen EU-Mitgliedstaaten aussagen können. Darin liegt ein großes Potenzial für die relativ stark auf, policy '-Analysen und institutionelle Fragestellungen fokussierte und gegenüber der gesellschaftlichen Konfliktdimension der europäischen Integration noch relativ desinteressierte Europaforschung. 